\title{
Ectopic ACTH syndrome caused by pulmonary carcinoid tumor mimicking long-standing sclerosing hemangioma
}

\author{
Sung Yong Han ${ }^{1}$, Bo Hyun Kim ${ }^{1,2}$, Hee Ryeong Jang ${ }^{1}$, Won Jin Kim ${ }^{1,2}$, Yun Kyung Jeon ${ }^{1,2}$, Sang Soo Kim², \\ and $\mathrm{In} \mathrm{Ju} \mathrm{Kim}^{1,2}$
}

${ }^{1}$ Department of Internal Medicine, ${ }^{2}$ Biomedical Research Insititute, Pusan National University Hospital, Busan, Korea
To the Editor,

Cushing syndrome is usually associated with a pituitary adenoma or adrenal adenoma. Ectopic adrenocorticotropic hormone (ACTH) secretion accounted for approximately 10\% of Cushing syndrome cases in several large series studies. It is difficult to distinguish between the 2 forms of ACTH-dependent Cushing syndrome Cushing disease and ectopic ACTH. The diagnosis is established by means of a bilateral inferior petrosal sinus sampling (IPSS) showing a lack of petrosal-to-peripheral ACTH gradient. Neuroendocrine tumors, especially small cell lung cancer and bronchial carcinoid tumors, are one of the causes of Cushing syndrome that is caused by ectopic ACTH secretion [1]. Pulmonary carcinoid tumors are also a cause of Cushing syndrome. Pulmonary carcinoid tumors are a rare cause of ectopic ACTH syndrome. The incidence of Cushing syndrome in pulmonary carcinoid tumors is approximately $1 \%$ [2].

We described a patient with ectopic ACTH syndrome stemming from a right middle lung nodule that remained unchanged over 7 years as a pulmonary carcinoid tumor mimicking sclerosing hemangioma.

A 45-year-old woman was admitted to our clinic with a past medical history of hypertension (9 months) and a typical cushingoid appearance, which had become apparent 6 months earlier. She had a $2 \mathrm{~cm}$ lung nodule, which was considered as a sclerosing hemangioma by radiologic and clinical findings. Computed tomography (CT) showed a well-defined nodule with mild enhancement. There was no calcification and fat component within the nodule. This solitary lung nodule had remained unchanged for 7 years (Fig. 1A). On physical examination, she had a moon face, central obesity, and diffuse ecchymoses on her entire body.

The results of basal endocrinological examinations were summarized in Table 1. Pituitary magnetic resonance imaging (MRI) scan was normal. CT scans of her chest, abdomen, and pelvis were normal except for the $2 \mathrm{~cm}$ lung nodule (Fig. 1B). Endoscopy and colonoscopy examinations were also conducted, but had nonspecific findings. She underwent a successful IPSS without complications, which revealed a petrosal/ peripheral ACTH ratio of 1.10 (Table 2). IPSS showed no central to peripheral ACTH gradient and lateralization. A positron emission tomography (PET)CT scan was performed and increased metabolic activity was detected on the 
Table 1. Results of endocrinological laboratory tests

\begin{tabular}{lcc}
\hline Variable & Result & Reference range \\
\hline Potassium, mmol/L & 2.47 & $3.5-5 \cdot 3$ \\
Adrenocorticotropic hormone, pg/mL & 251.68 & $10-60$ \\
Cortisol, $\mu \mathrm{g} / \mathrm{dL}$ & $>50.0$ & $5-25$ \\
$24 \mathrm{hr}$ urine free-cortisol, $\mu \mathrm{g} / \mathrm{day}$ & $1,033.7$ & $21-85$ \\
After low-dose dexamethasone suppression test cortisol, $\mu \mathrm{g} / \mathrm{dL}$ & 37.1 & 24.1 \\
After high-dose dexamethasone suppression test cortisol, $\mu \mathrm{g} / \mathrm{dL}$ & Negative & Negative \\
Urine 5-hydroxyindoleacetic acid & 3.6 & $1.8-7.5$ \\
Plasma serotonin, ng/mL & 3.17 & $0-10$ \\
Calcitonin, $\mathrm{mg} / \mathrm{mL}$ & & \\
\hline
\end{tabular}
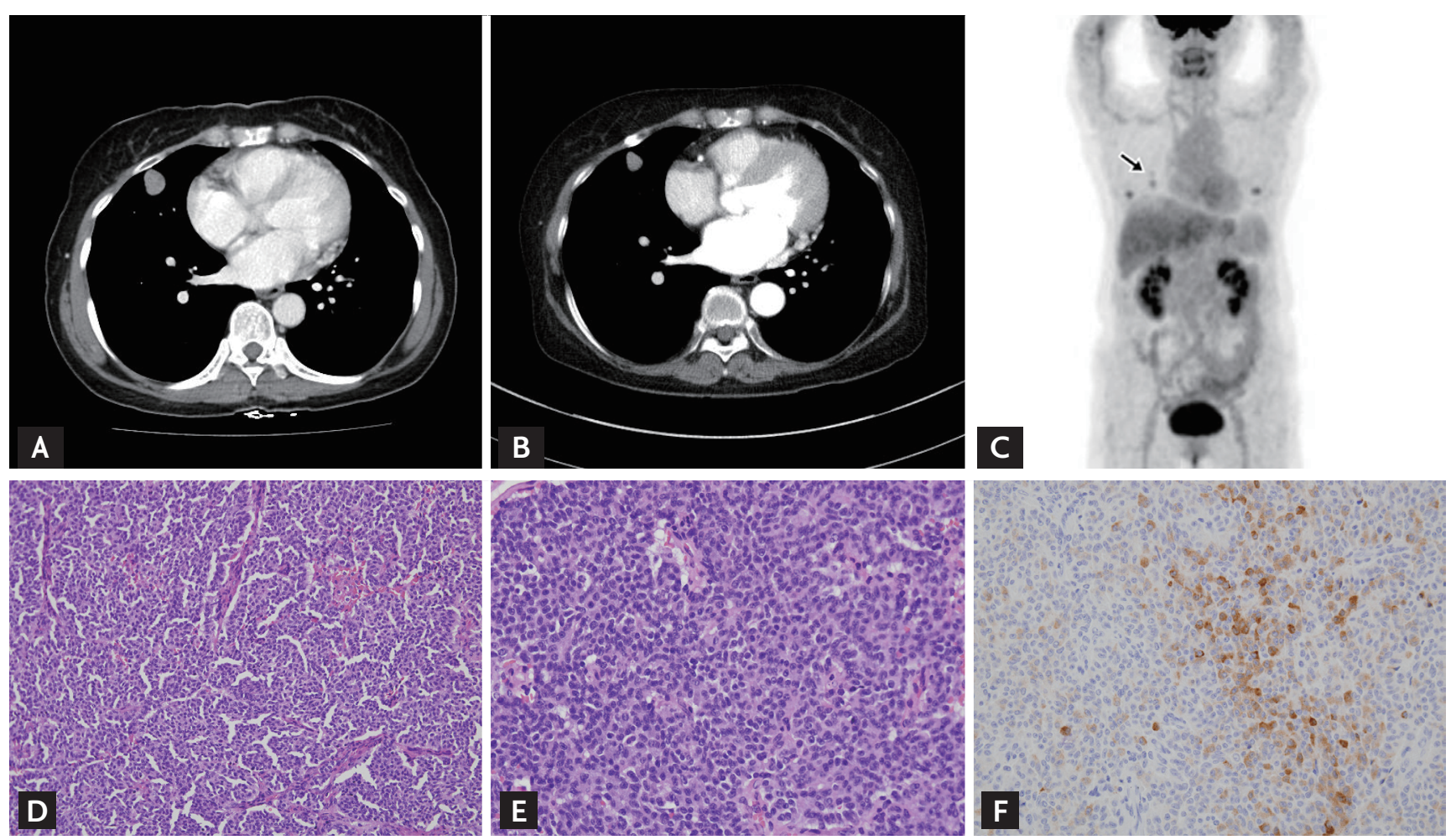

Figure 1. (A) Chest computed tomography (CT) axial image with contrast enhancement shows a well-defined nodule measuring $2 \mathrm{~cm}$ in right middle lobe. There is no calcification and fat component in the nodule (September 2007). (B) In July 2014, chest CT axial image with contrast enhancement display a nodule in right middle lobe. There is no change of nodule size between two CT image. (C) Fused positron emission tomography (PET)/CT image show a nodule with increased 18F-fluorodeoxyglucose uptake (maximum standardized uptake value, 1.7) in right middle lobe (arrow). Histological features of lung mass. H\&E stained section of a typical pulmonary carcinoid (D, ×200; E, ×400). (F) Positive immunohistochemical stain for adrenocorticotropic hormone (x400).

lung nodule (maximum standardized uptake value, 1.7) (Fig. 1C).

We concluded that the ectopic ACTH syndrome was associated with the lung nodule and decided to perform lung nodule biopsy. However, the patient did not con- sent due to the bleeding risk from the hemangioma and only agreed to resection of the lung lesion. She accordingly received a wedge resection with video-assisted thoracoscopic surgery. Histology showed a typical carcinoid tumor. Immunohistochemistry stains were positive for 
Table 2. Results of inferior petrosal sinus sampling

\begin{tabular}{lccc}
\hline & \multirow{2}{*}{ Peripheral ACTH, pg/mL } & \multicolumn{2}{c}{ Petrosal ACTH (petrosal/peripheral ACTH ratio) } \\
\cline { 3 - 4 } & 214.3 & Right, pg/mL & Left, pg/mL \\
\hline Basal & 233.8 & $227.1(1.06)$ & $235.8(1.10)$ \\
Peak & & $241.5(1.03)$ & $260.9(1.12)$ \\
\hline
\end{tabular}

ACTH, adrenocorticotropic hormone.

ACTH, CD56, synaptophysin, and thyroid transcription factor-1 (Fig. 1D-1F). Following resection of lung lesion, plasma levels of ACTH and cortisol decreased significantly (preoperative ACTH $251.69 \mathrm{pg} / \mathrm{mL}$, cortisol > 50.0 $\mu \mathrm{g} / \mathrm{dL}$; postoperative ACTH $5.59 \mathrm{pg} / \mathrm{mL}$, cortisol $1.76 \mu \mathrm{g} /$ $\mathrm{dL})$. She experienced general weakness post-surgery, and moon face progressively resolved. She received replacement steroid i.e., hydrocortisone $10 \mathrm{mg}$ in the morning and $5 \mathrm{mg}$ in the evening. Three month after surgery, we rechecked ACTH/cortisol (ACTH, $2.84 \mathrm{pg} / \mathrm{mL}$; cortisol, $0.32 \mu \mathrm{g} / \mathrm{dL}$ ). She no longer felt general weakness.

The association between cancer and Cushing syndrome was first recognized in 1928 , in a patient with small cell carcinoma of the lung. The definition of this disease as a syndrome is attributed to Meador et al. [3] who, in 1962, demonstrated the biological activity of ACTH in pulmonary carcinoid tumors.

Pulmonary carcinoid tumors arise from neuroendocrine Kulchitsky cells located in the bronchial epithelium and comprise between $2 \%$ to $5 \%$ of all primary lung cancers [4]. About $25 \%$ of lung carcinoid tumors are located within the airways and are referred to as bronchial carcinoids. Pulmonary carcinoid tumors are not located within the airway. Ectopic ACTH secretion accounts for approximately $10 \%$ of Cushing syndrome. The common causes of ectopic ACTH include small cell lung carcinoma (about $27 \%$ ), bronchial carcinoids (21\%), islet cell tumors of the pancreas (16\%), and thymic carcinoids (10\%). Pulmonary carcinoids typically have a long history and slow onset of symptoms (1 to 84 months; median, 23.6), and cushingoid features are usually present [5].

Frequent signs of hypercortisolism are weakness, hypertension, glucose intolerance, hypokalemia, alkalosis, weight loss, anemia, and hyperpigmentation. The typical features of Cushing syndrome are more frequent in slowly progressing tumors.

Well-differentiated pulmonary neuroendocrine tumors are usually indolent, with metastases reported in
$<15 \%$ of cases. The presence of lymph node metastases and the presence of symptoms at the time of diagnosis are adverse prognostic factors. Most studies have found 5-year survival rates of $>90 \%$.

Management of patients with ectopic ACTH requires control of the hypercortisolemia as soon as the diagnosis is established. Tumors resection is indicated in patients with identifiable sources of ectopic ACTH. Surgery may be curative in $>80 \%$ of pulmonary carcinoids. Surgical removal has resulted in lower rates of recurrence and excellent long-term survival.

The lung nodule was considered as a sclerosing hemangioma by the radiologist and the patient did not have any symptoms of cortisol excess. Seven years later, the patient had symptoms of cortisol excess for at least 6 months prior to presentation. Laboratory results showed that she had ectopic Cushing syndrome. Diagnostic tests for the ectopic ACTH syndrome, including a chest CT all had normal findings, except for the lung nodule.

Lung lesions are the most common cause of ectopic ACTH syndrome. However, in this case, the size of the lung nodule was unchanged for 7 years. It is generally acknowledged that even a slow growing tumor changes size within a couple of years, hence we did not initially consider it as the cause of ectopic ACTH syndrome. However, in this case, no other possible etiological lesions of ectopic ACTH were detected. We therefore considered the possibility that the ectopic ACTH syndrome was associated with the lung nodule. Plasma ACTH decreased significantly post lesional resection.

The case demonstrated that a largely unchanged lung nodule is a possible cause of ectopic ACTH syndrome. Diagnosis of Cushing syndrome must consider lung nodular lesions. Furthermore, if the lung nodule is a benign lung hemangioma, we recommend that physicians not exclude it as the etiology of Cushing syndrome.

Keywords: ACTH syndrome, ectopic; Carcinoid tumor; 
Neuroendocrine tumors

\section{Conflict of interest}

No potential conflict of interest relevant to this article was reported.

\section{REFERENCES}

1. Kulke MH, Mayer RJ. Carcinoid tumors. N Engl J Med 1999;340:858-868.

2. de Matos LL, Trufelli DC, das Neves-Pereira JC, Danel C, Riquet M. Cushing's syndrome secondary to bron- chopulmonary carcinoid tumor: report of two cases and literature review. Lung Cancer 2006;53:381-386.

3. Meador CK, Liddle GW, Island DP, et al. Cause of Cushing's syndrome in patients with tumors arising from "nonendocrine" tissue. J Clin Endocrinol Metab 1962;22:693-703.

4. Deb SJ, Nichols FC, Allen MS, Deschamps C, Cassivi SD, Pairolero PC. Pulmonary carcinoid tumors with Cushing's syndrome: an aggressive variant or not? Ann Thorac Surg 2005;79:1132-1136.

5. Beuschlein F, Hammer GD. Ectopic pro-opiomelanocortin syndrome. Endocrinol Metab Clin North Am 2002;31:191-234. 